hep-th/0306152v4

\title{
Statistical Mechanics of Three-dimensional Kerr-de Sitter Space
}

\author{
Mu-In Park ${ }^{1}$ \\ Research Institute of Physics and Chemistry, \\ Chonbuk National University, Chonju 561-756, Korea
}

\begin{abstract}
The statistical entropy computation of the $(2+1)$-dimensional Kerr-de Sitter space in the context of the classical Virasoro algebra for an asymptotic isometry group has been a mystery since first, the degeneracy of the states has the right value only at the infinite boundary which is casually disconnected from our universe, second, the analyses were based on the unproven Cardy's formula for complex central charge and conformal weight. In this paper, I consider the entropy in Carlip's "would-be gauge" degrees of freedom approach instead. I find that it agrees with the Bekenstein-Hawking entropy but there are no the above problems. Implications to the dS/CFT are noted.
\end{abstract}

PACS Nos: 04.60.-m, 04.60.-Kz, 11.40.-q.

Keywords: dS/CFT, Chern-Simons, Statistical entropy.

06 Feb. 2009

\footnotetext{
1 Electronic address: muinpark@yahoo.com
} 
After recent cosmological observations that suggest the existence of a positive cosmological constant $\Lambda$ there have been extensive studies on the de-Sitter space. Especially, in the context of $\mathrm{dS} / \mathrm{CFT}$ proposal $[1,2,3,4]^{2}$, there have been increasing interests [5] in statistical computation of the Bekenstein-Hawking $(\mathrm{BH})$ entropy for the three-dimensional Kerr-de Sitter $\left(K d S_{3}\right)$ space $[6]$

$$
S=\frac{2 \pi r_{+}}{4 \hbar G}
$$

from the "classical" Virasoro algebra, associated with an asymptotic isometry group at the infinite boundary à la Strominger and Birmingham [7]. Here, $r_{+}$is the radius of the cosmological horizon. But, there have been two problems in those analyses. The first is a conceptual problem that the entropy was computed at the infinite boundary which is casually disconnected from the observer who is sitting in our universe, the interior $\left(r<r_{+}\right)$of the cosmological horizon. The second is that the analyses were based on a plausible but unproven assumption about the extended Cardy's formula [8] for the complex central charge and conformal weight. (See [9] and [10] for recent strugglings with this problem.)

In this paper I consider the entropy in the context of Carlip's "would-be gauge" degrees of freedom approach [11] instead and show that the above two problems are resolved. The use is made of $S L(2, \mathbf{C})$ WZW theory on the horizon and its associated well-defined Virasoro operator. The obtained entropy agrees with the BH entropy (1) but there are no the problems above. Implications to the dS/CFT are noted also.

The (2+1)-dimensional gravity for a cosmological constant $\Lambda=1 / \ell^{2}$ is described by the action

$$
I_{g}=\frac{1}{16 \pi G} \int d^{3} x \sqrt{-g}\left(R-\frac{2}{\ell^{2}}\right)+I_{m}
$$

where $I_{m}$ is the matter action [the details are not important in this paper] and I have omitted the surface terms in the pure gravity part. This theory has a constant curvature $R=2 \ell^{-2}$, outside the matters.

For the positive cosmological constant, i.e., real valued $\ell$, (2) has the Kerr-type vacuum solution, called three-dimensional Kerr-de Sitter $\left(K d S_{3}\right)$, (from now on, I take $G=1 / 8$ unless otherwise stated)

$$
d s^{2}=-\left(M-\frac{r^{2}}{\ell^{2}}+\frac{J^{2}}{4 r^{2}}\right) d t^{2}+\left(M-\frac{r^{2}}{\ell^{2}}+\frac{J^{2}}{4 r^{2}}\right)^{-1} d r^{2}+r^{2}\left(-\frac{J}{2 r^{2}} d t+d \varphi\right)^{2}
$$

\footnotetext{
${ }^{2}$ Ref. [1] provides the first explicit example of the dS/CFT in the three-dimensional Kerr-de Sitter space. In Ref. [2], as the direct consequence of Ref. [1], "dS/CFT" for the three-dimensional case was noted explicitly for the first time: See, for example, below Eq. (47).
} 
with the mass and angular momentum parameters $M$ and $J$, respectively $[1,4,9,10,12,13]$; $\varphi$ has period $2 \pi$. This solution has a cosmological horizon at

$$
r_{+}=\frac{\ell}{\sqrt{2}} \sqrt{M+\sqrt{M^{2}+\frac{J^{2}}{\ell^{2}}}}
$$

with no bound on $M$ and $J$. By introducing $r_{(-)} \equiv(\ell / \sqrt{2}) \sqrt{-M+\sqrt{M^{2}+J^{2} / \ell^{2}}}$, the metric (3) can be conveniently written in the proper radial coordinates as

$$
d s^{2}=\sinh ^{2} \rho\left(\frac{r_{+} d t}{\ell}-r_{(-)} d \varphi\right)^{2}-\ell^{2} d \rho^{2}+\cosh ^{2} \rho\left(\frac{r_{(-)} d t}{\ell}+r_{+} d \varphi\right)^{2}
$$

with

$$
\begin{gathered}
M=\frac{r_{+}^{2}-r_{(-)}^{2}}{\ell^{2}}, J=\frac{2 r_{+} r_{(-)}}{\ell}, \\
r^{2}=r_{+}^{2} \cosh ^{2} \rho+r_{(-)}^{2} \sinh ^{2} \rho .
\end{gathered}
$$

In these coordinates, the cosmological event horizon is at $\rho=0$, and the exterior $(r>$ $\left.r_{+}\right)$/interior $\left(r<r_{+}\right)$of the horizon is represented by real/imaginary value $\rho$. The interior and exterior regions are casually disconnected and the horizon acquires the thermodynamic entropy (1). I seek a statistical derivation of (1) in the context of the $(2+1)$-dimensional quantum gravity with would-be gauge degrees of freedom "on the horizon", à la Carlip [11].

The quantization of (2) is best achieved by recasting the theory as a $S L(2, \mathbf{C})$ Chern-Simons gauge theory $[14,15]$. The action for this theory is, up to surface terms,

$$
I_{g}[A]=\frac{i s}{4 \pi} \int_{D_{2} \times R} d^{3} x \epsilon^{\mu \nu \rho}\left\langle A_{\mu} \partial_{\nu} A_{\rho}+\frac{2}{3} A_{\mu} A_{\nu} A_{\rho}\right\rangle-\frac{i s}{4 \pi} \int_{D_{2} \times R} d^{3} x \epsilon^{\mu \nu \rho}\left\langle\bar{A}_{\mu} \partial_{\nu} \bar{A}_{\rho}+\frac{2}{3} \bar{A}_{\mu} \bar{A}_{\nu} \bar{A}_{\rho}\right\rangle
$$

on the manifold $\Sigma=D_{2} \times R$, where $D_{2}$ is a 2-dimensional disc of the space at a constant time $t$, with its boundary $\partial D_{2}$ at the cosmological horizon $r_{+} ; A_{\mu}$ is an $S L(2, \mathbf{C})$ gauge field and $\bar{A}_{\mu}$ is its complex conjugate; $\langle\cdots\rangle$ denotes the trace. (7) is equivalent to (2) with the identification 3

$$
A_{\mu}^{a}=\omega_{\mu}^{a}+\frac{e_{\mu}^{a}}{i \ell}, \bar{A}_{\mu}^{a}=\omega_{\mu}^{a}-\frac{e_{\mu}^{a}}{i \ell} \quad(a=0,1,2)
$$

with

$$
s=2 \ell .
$$

3 I use the same conventions as Ref. [1] $: t_{0}=\frac{1}{2}\left(\begin{array}{cc}0 & -1 \\ 1 & 0\end{array}\right), t_{1}=\frac{1}{2}\left(\begin{array}{cc}1 & 0 \\ 0 & -1\end{array}\right), t_{2}=\frac{1}{2}\left(\begin{array}{ll}0 & 1 \\ 1 & 0\end{array}\right)$ such that $\left[t_{a}, t_{b}\right]=\epsilon_{a b}{ }^{c} t_{c}$ and $\left\langle t_{a} t_{b}\right\rangle=\frac{1}{2} \eta_{a b}$, where $\epsilon_{012}=1$ and $\eta_{a b}=\operatorname{diag}(-1,1,1)$. The metric $\eta_{a b}$ is used to raise and lower indices. 
Here, $e^{a}=e_{\mu}^{a} d x^{\mu}, \omega^{a}=\frac{1}{2} \epsilon^{a b c} \omega_{\mu b c} d x^{\mu}$ are the triads and the $S L(2, \mathbf{R})$ spin connections, respectively. It is easily checked that the $K d S_{3}$ solution (3) is represented by

$$
\begin{aligned}
& \mathbf{A}^{0}=-\frac{r_{+}+i r_{(-)}}{\ell}\left(\frac{d t}{\ell}+i d \varphi\right) \sinh \rho, \\
& \mathbf{A}^{1}=d \rho, \\
& \mathbf{A}^{2}=-\frac{r_{+}+i r_{(-)}}{\ell}\left(\frac{d t}{\ell}+i d \varphi\right) \cosh \rho,
\end{aligned}
$$

where the superscript indices denote the group indices $a=0,1,2$. From this, the polar components are obtained as

$$
A_{\rho}=t_{1}, A_{\varphi}=-i z\left(U^{-1} t_{2} U\right), A_{t}=i A_{\varphi}
$$

where

$$
z=\left(r_{+}+i r_{(-)}\right) / \ell, \quad U=\left(\begin{array}{cc}
e^{\rho / 2} & 0 \\
0 & e^{-\rho / 2}
\end{array}\right)
$$

This insures that there is asymptotic isometries as $\rho \rightarrow \infty$ and its associated ( two-copies of ) "classical" Virasoro algebra [1]. The obtained statistical entropy coincides with (1), which means that the appropriate CFT which gives the correct entropy of a horizon would live at the infinite boundary, as coincides exactly with Strominger's dS/CFT proposal [4]. But, in the following, I will show that (11) also allows to acquire the BH entropy (1) from a quantum mechanical analysis of $S L(2, \mathbf{C})$ WZW conformal field theory on the horizon $r_{+}$which is located at the space boundary $\partial D_{2}$, by a direct adaptation of Carlip's analysis [11].

To this end, I first note that the gauge connection (11) satisfies the boundary conditions for a black hole horizon $r_{+}$, even if $r_{(-)}$is an arbitrary real parameter, $\tilde{r}_{(-)}$[16]. This means that we have to sum over its possible values to count macroscopically in-distinguishable states [11]. In the following I will find the value of $\tilde{r}_{(-)}$which gives the dominant contributions to the entropy.

Imposing the Gauss' law constraint $F_{i j}^{a}=0$ reduces $(7)$ to a $S L(2, \mathbf{C})$ chiral WZW action $[1,17,18,19]$, up to the surface terms which vanish on the horizon $r_{+}[16]$,

$$
\begin{aligned}
I_{g}[A] & =I_{C W Z W}[A]-I_{C W Z W}[\bar{A}] \\
I_{C W Z W}[A] & =-\frac{i s}{4 \pi} \int_{D_{2}} \epsilon_{i j}\left\langle A_{i} A_{j} A_{0}\right\rangle+\frac{i s}{4 \pi} \oint_{\partial D_{2}} d \varphi\left\langle A_{\varphi} A_{0}\right\rangle
\end{aligned}
$$

with

$$
A_{\mu}=g^{-1} \partial_{\mu} g
$$


The Poisson bracket for $A_{\varphi}^{a}$, which lives on $\partial D_{2}=r_{+}$, is computed [20] as

$$
\left\{A_{\varphi}^{a}(\varphi), A_{\varphi}^{b}\left(\varphi^{\prime}\right)\right\}=\frac{4 \pi}{i s} \epsilon^{a b}{ }_{c} A_{\varphi}^{c}(\varphi) \delta\left(\varphi-\varphi^{\prime}\right)+\frac{4 \pi}{i s} \eta^{a b} \partial_{\varphi} \delta\left(\varphi-\varphi^{\prime}\right)
$$

which is the $S L(2, \mathbf{C})$ Kac-Moody algebra in the density form $[1,17,18]$. The current operators $J_{n}^{a}, \bar{J}_{n}^{a}$ which are defined by

$$
A_{\varphi}^{a}=\frac{2}{i s \hbar} \sum_{n=-\infty}^{\infty} J_{n}^{a} e^{i n \varphi}, \quad{\overline{A_{\varphi}}}^{a}=-\frac{2}{i s \hbar} \sum_{n=-\infty}^{\infty} \bar{J}_{n}^{a} e^{i n \varphi}
$$

satisfy $S L(2, \mathbf{C})$ operator current algebra

$$
\begin{aligned}
{\left[J_{m}^{a}, J_{n}^{b}\right] } & =i \epsilon_{c}^{a b} J_{m+n}^{c}+k m \eta^{a b} \delta_{m+n, 0} \\
{\left[J_{m}^{a}, \bar{J}_{n}^{b}\right] } & =0 \\
{\left[\bar{J}_{m}^{a}, \bar{J}_{n}^{b}\right] } & =i \epsilon_{c}^{a b} \bar{J}_{m+n}^{c}-k m \eta^{a b} \delta_{m+n, 0}
\end{aligned}
$$

with the imaginary level

$$
k=-\frac{i s}{2 \hbar}
$$

The Virasoro operators $L_{0}, \bar{L}_{0}$ for the action (7) are then given by the Sugawara construction $[21]$ as

$$
L_{0}=\frac{1}{2 k+Q} \sum_{n=-\infty}^{\infty}\left\langle: J_{-n} J_{n}:\right\rangle^{\prime}, \quad \bar{L}_{0}=\frac{1}{-2 k+Q} \sum_{n=-\infty}^{\infty}\left\langle: \bar{J}_{-n} \bar{J}_{n}:\right\rangle^{\prime},
$$

which satisfy

$$
\left[L_{0}, J_{n}^{a}\right]=-n J_{n}^{a}, \quad\left[\bar{L}_{0}, \bar{J}_{n}^{a}\right]=-n \bar{J}_{n}^{a} .
$$

Here $\langle J J\rangle^{\prime}=\eta_{a b} J^{a} J^{b}$ and $Q \eta^{a d}=\epsilon_{b c}^{a} \epsilon^{d b c} / 4=-(1 / 2) \eta^{a d}$ is the quadratic Casimir in the adjoint representation. Writing the nonzero mode piece as level number $N, L_{0}+\bar{L}_{0}$ becomes

$$
\begin{aligned}
L_{0}+\bar{L}_{0} & =N-\left(\frac{i s}{\hbar}+\frac{1}{2}\right)^{-1}\left\langle J_{0} J_{0}\right\rangle^{\prime}+\left(\frac{i s}{\hbar}-\frac{1}{2}\right)^{-1}\left\langle\bar{J}_{0} \bar{J}_{0}\right\rangle^{\prime} \\
& =N-\left(\frac{s^{2}}{\hbar^{2}}+\frac{1}{4}\right)^{-1} \frac{s^{2}}{4 \hbar^{2} \ell^{2}}\left(\frac{4 s}{\hbar} r_{+} \tilde{r}_{(-)}+r_{+}^{2}-\tilde{r}_{(-)}^{2}\right),
\end{aligned}
$$

where I have used the relations, from (11) and (16),

$$
\begin{aligned}
\left\langle J_{0} J_{0}-\bar{J}_{0} \bar{J}_{0}\right\rangle^{\prime} & =\frac{s^{2}}{\hbar^{2}} \frac{i r_{+} \tilde{r}_{(-)}}{\ell^{2}} \\
\left\langle J_{0} J_{0}+\bar{J}_{0} \bar{J}_{0}\right\rangle^{\prime} & =\frac{s^{2}}{2 \hbar^{2}} \frac{r_{+}^{2}-\tilde{r}_{(-)}^{2}}{\ell^{2}}
\end{aligned}
$$


For non-horizon boundaries, the Virasoro constraints become $L_{m}=0, \bar{L}_{m}=0(m \geq 0)$ with

$$
L_{m} \equiv \frac{1}{2 k+Q} \sum_{n=-\infty}^{\infty}\left\langle: J_{m-n} J_{n}:\right\rangle^{\prime}, \quad \bar{L}_{m} \equiv \frac{1}{-2 k+Q} \sum_{n=-\infty}^{\infty}\left\langle: \bar{J}_{m-n} \bar{J}_{n}:\right\rangle^{\prime}
$$

[21] (for some more general expressions, see Ref. [22]) and $\mathcal{H} \equiv L_{m}-\bar{L}_{m}, \mathcal{H}_{\varphi} \equiv L_{m}+\bar{L}_{m}$ are the diffeomorphism generators (in the momentum space) along $t$ and $\varphi$, respectively. However, for the horizon boundaries, where the lapse function-the Lagrange multiplier for $\mathcal{H}$-vanishes, $\mathcal{H}$ is not constrained to be zero. Moreover, from the constancy of the (angular) shift function-the multiplier for $\mathcal{H}_{\varphi}$-along $\varphi$, only $L_{0}+\bar{L}_{0}=0$, which does not depends on $\varphi$, can be implemented $[11,16]$.

From the remnant of the (quantum) constraint equation $L_{0}+\bar{L}_{0}=0$, which corresponds to the Wheeler-de Witt equation [11], on the horizon $\partial D_{2}=r_{+}$, one obtains as

$$
N=\frac{s^{2}}{\hbar^{2} \ell^{2}} r_{+}^{2}-4 \ell^{2}\left(1+\frac{\hbar^{2}}{4 s^{2}}\right)^{-1}\left(\tilde{r}_{(-)}-\frac{2 s}{\hbar} r_{+}\right)^{2} \text {. }
$$

Up to now, the real parameter $\tilde{r}_{(-)}$is arbitrary and so we should sum over its possible values to count the number of macroscopically indistinguishable states. However, in order to compute the dominant contribution of $N$ to the number of states $\Omega \approx \exp (2 \pi \sqrt{c N / 6})$ [8, 23], it is enough to know its maximum value $N_{\max }$, which is attained as

$$
N_{\max }=\frac{s^{2}}{\hbar^{2} \ell^{2}} r_{+}^{2}=\left(\frac{2 r_{+}}{\hbar}\right)^{2}
$$

for $\tilde{r}_{(-)}=(2 s / \hbar) r_{+}$, in our convention (9). In the semiclassical regime of large $s$, i.e., small $\Lambda$, the central charge may be approximated by that of six - three $(a=1,2,3)$ for each Kac-Moody sector and there are two Kac-Moody sectors with the currents $J_{n}^{a}, \bar{J}_{n}^{a}$-independent bosonic oscillators such that $c \approx 6[11,24]$. Then the dominant contrbution to the statistical entropy, through the Cardy's formula $[8,23,24]$ for the asymptotic density of states of a conformal field theory, is

$$
S_{\mathrm{st}}=\ln \Omega \approx 2 \pi \sqrt{\frac{c N_{\max }}{6}}=\frac{4 \pi r_{+}}{\hbar} .
$$

This agrees completely with the thermodynamic entropy (1), after recovering the Newton's constant $G$. Here note that the correct $1 / \hbar$ factor, whose origin has not been explicit in the literatures (see [16] for a comparison), comes from the definition (17), essentially. (26) is the same result as in the negative $\Lambda$, i.e., BTZ black hole case [16], which can be recaptured by $\ell \rightarrow i \ell, s \rightarrow i s, \tilde{r}_{(-)} \rightarrow i \tilde{r}_{-}:$The crucial point is that (24) is invariant under this substitution, 
neglecting the sign change of $\left(1-\hbar^{2} / 4 s^{2}\right)^{-1}$ which is not important in the computation of $N_{\max }$; a similar analysis for the de-Sitter vacuum $(M=1, J=0)$ has been studied by Strominger and Maldacena [25] several years ago in the context of Carlip's boundary conditions [11], but this property has not been evident in their approach.

To conclude, two remarks are in order.

First, unlike the analyses of the asymptotic isometries [1], the Cardy's formula (26) is well defined with the real valued $c$ and $N_{\max }$. It is essential to restrict to the horizon in order to acquire the correct $\mathrm{BH}$ entropy. ${ }^{4}$

Second, note that maximum value of $N$ comes from the $Q$ term essentially which is purely quantum effect. ${ }^{5}$ Without that term, there is no $r_{+}^{2}-\tilde{r}_{(-)}^{2}$ term in $(21)$ such that there are no extremal points of $N$ which satisfies $L_{0}+\bar{L}_{0}=0$; rather, it increases indefinitely as $N=$ $(s / 2 \hbar) r_{+} \tilde{r}_{(-)} / \ell^{2}$ which has no saddle points so that the Cardy's formula (26) can not be applied. This is sharply contrast to the analysis of the asymptotic isometries of (11), where the "classical" central charges in the (two-copies of ) Virasoro algebra has been crucial to obtain the correct BH entropy and additional quantum corrections gives only some small corrections to (1) [28]. Hence, I have two dual descriptions of black hole/cosmological horizons. One is described by the two-copies of classical Virasoro algebra at the infinite boundary and the other's by one-copy of the quantum Virasoro generator through the Sugawara construction at the horizon $r_{+}$. By combining the Strominger's dS/CFT proposal and the horizon holography [31] one can expect that the two descriptions would be closely related. It would be an outstanding challenge to find a direct connection, or a $R G$-flow, of these two dual descriptions. ${ }^{6}$ This will shed some light on the mystery of dS/CFT correspondence.

\section{Acknowledgments}

This work was supported by the Korean Research Foundation Grant funded by Korea Government(MOEHRD) (KRF-2007-359-C00011).

\section{References}

[1] M.-I. Park, Phys. Lett. B 440 (1998) 275 [hep-th/9806119].

[2] M.-I. Park, Nucl. Phys. B 544 (1999) 377 [hep-th/9811033].

\footnotetext{
${ }^{4}$ Similar result has been obtained in different classical contexts [26, 27, 28, 29].

5 This seems to be in contrast to a recent analysis in a different polarization [30]. But the status of the Cardy's formula for this polarization remains unclear.

${ }^{6}$ After writing this paper, I became aware that Sach and Solodukhin also have presented a similar idea [32].
} 
[3] C. M. Hull, JHEP 9807 (1998) 021 [hep-th/9806146]; R. Bousso, JHEP 9906 (1999) 028 [hep-th/9906022]; V. Balasubramanian, P. Horava and D. Minic, JHEP 0105 (2001) 043 [hep-th/0103171]; E. Witten, hep-th/0106109; E. Silverstein, hep-th/0106209.

[4] A. Strominger, JHEP 0110 (2001) 034 [hep-th/0106113]; M. Spradlin, A. Strominger, and A. Volovich, hep-th/0110007.

[5] S. Nojiri and S. D. Odintsov, Phys. Lett. B 519 (2001) 145 [hep-th/0106191]; D. Klemm, Nucl. Phys. B 625 (2002) 295 [hep-th/0106247]; V. Balasubramanian, J. de Boer, and D. Minic, Phys. Rev. D 65 (2002) 123508 [hep-th/0110108].

[6] G. W. Gibbons and S. W. Hawking, Phys. Rev. D 15 (1977) 2738.

[7] A. Strominger, JHEP 9802 (1998) 009 [hep-th/9712251]; D. Birmingham, I. Sachs, and S. Sen, Phys. Lett. B 424 (1998) 275 [hep-th/9801019]; M.-I. Park, Phys. Lett. B 597 (2004) 237 [hep-th/0403089].

[8] J. A. Cardy, Nucl. Phys. B 270 (1986) 186.

[9] R. Bousso, A. Maloney and A. Strominger, Phys. Rev. D 65 (2002) 104039 [hepth/0112218]; M. Spradlin and A. Volovich, Phys. Rev. D 65, 104037 (2002) [hepth/0112223]; E. Buffenoir, K. Noui and P. Roche, Class. Quant. Grav. 19 (2002) 4953 [hep-th/0202121]; V. Balasubramanian, J. de Boer, and D. Minic, Class. Quant. Grav. 19 (2002) 5655 [hep-th/0207245]; J. Kluson, Class. Quant. Grav. 20 (2003) 2131 [hepth/0302008].

[10] M.-I. Park, Class. Quant. Grav. 25 (2008) 135003 [arXiv:0705.4381].

[11] S. Carlip, Phys. Rev. D 51 (1995) 632.

[12] S. Deser and R. Jackiw, Ann. Phys. 153 (1984) 405.

[13] Y. S. Myung, Mod. Phys. Lett. A 16 (2001) 2353 [hep-th/0110123].

[14] A. Achúcaro and P. K. Townsend, Phys. Lett. B 180 (1986) 89; E. Witten, Nucl. Phys. B 311 (1988) 46.

[15] E. Witten, Commun. Math. Phys. 137 (1991) 29.

[16] M. Bañados and A. Gomberoff, Phys. Rev. D 55 (1997) 6162 [gr-qc/9611044].

[17] G. Moore and N. Seiberg, Phys. Lett. B 220 (1989) 422; S. Elitzur, G. Moore, A. Schwimer and N. Seiberg, Nucl. Phys. B 326 (1989) 108. 
[18] P. Oh and M.-I. Park, Mod. Phys. Lett. A 14 (1999) 231 [hep-th/9805178]; P. Oh, Phys. Lett. B 464 (1999) 19.

[19] J. Fjelstad and S. Hwang, Phys. Lett. B 466 (1999) 227 [hep-th/9906123].

[20] E. Witten, Commun. Math. Phys. 92 (1984) 455.

[21] P. Di Francesco, P. Mathieu, and D. Sénéchal, Conformal Field Theory (Springer, New York, 1997).

[22] M. Banados, Phys. Rev. D 52 (1996) 5816 [arXiv:hep-th/9405171].

[23] M.-I. Park, JHEP 12 (2004) 041 [hep-th/0402173]; Phys. Rev. D 77 (2008) 026011 [hepth/0608165]; Phys. Rev. D 77 (2008) 126012 [hep-th/0609027].

[24] K. Huang and S. Weinberg, Phys. Rev. Lett. 25 (1970) 895.

[25] J. Maldacena and A. Strominger, JHEP 9802 (1998) 014 [gr-qc/9801096].

[26] S. Carlip, Phys. Rev. Lett. 82 (1999) 2828 [hep-th/9812013].

[27] M.-I. Park and J. Ho, Phys. Rev. Lett. 83 (1999) 5595 [hep-th/9910158]; M.-I. Park and J. H. Yee, Phys. Rev. D 61 (2000) 088501 [9910213].

[28] M.-I. Park, Nucl. Phys. B 634 (2002) 339 [hep-th/0111224].

[29] G. Kang, J.-I. Koga, and M.-I. Park, Phys. Rev. D 70 (2004) 024005 [hep-th/0402113].

[30] J. Fjelstad, S. Hwang, and T. Mansson, Nucl. Phys. B 641 (2002) 376 [hep-th/0206113].

[31] G. 't Hooft, in Salam Fest (World Scientific Co. Singapore, 1993) [gr-qc/9310026]; L. Susskind, J. Math. Phys. 36 (1995) 6377.

[32] I. Sachs and S. N. Solodukhin, Phys. Rev. D 64 (2001) 124023 [hep-th/0107173]. 